\title{
Dense, Unspecific Connectivity of Neocortical Parvalbumin-Positive Interneurons: A Canonical Microcircuit for Inhibition?
}

\author{
Adam M. Packer and Rafael Yuste \\ HHMI, Department of Biological Sciences, Columbia University, New York, New York 10027
}

\begin{abstract}
GABAergic interneurons play a major role in the function of the mammalian neocortex, but their circuit connectivity is still poorly understood. We used two-photon RuBi-Glutamate uncaging to optically map how the largest population of cortical interneurons, the parvalbumin-positive cells $(\mathrm{PV}+)$, are connected to pyramidal cells $(\mathrm{PCs})$ in mouse neocortex. We found locally dense connectivity from PV + interneurons onto PCs across cortical areas and layers. In many experiments, all nearby PV + cells were connected to every local PC sampled. In agreement with this, we found no evidence for connection specificity, as PV + interneurons contacted PC pairs similarly regardless of whether they were synaptically connected or not. We conclude that the microcircuit architecture for PV + interneurons, and probably neocortical inhibition in general, is an unspecific, densely homogenous matrix covering all nearby pyramidal cells.
\end{abstract}

\section{Introduction}

The mammalian neocortex is a marvel of biological engineering capable of impressive computational feats. This structure appears to be organized in a stereotypical and hierarchical manner, presumably to achieve a high level of parallel processing power (Mountcastle, 1982). Unfortunately, the structure of cortical microcircuits appears to be an "impenetrable jungle" (Ramón y Cajal, 1923) because of the dense mixing of different types of neurons, which makes deducing the way it operates difficult. Indeed, although cortical preparations have been studied anatomically and physiologically for over a century, remarkably little has been revealed about the fine-scale organization of these neurons into circuits. It is clear that the cortex is divisible into layers and areas, each with distinct connectivity patterns with other layers and areas, presumably resulting in specific functions for each element (Douglas and Martin, 2004). It is also known that individual neurons belong to particular classes, although there is still no valid classification for many neurons (Ascoli et al., 2008) or theoretical framework describing how they function in a concerted way to process information.

Part of the reason for this ignorance is the experimental difficulty in revealing how these neurons are connected to each other to form functional circuits (Crick, 1979). In the last decade, several new tech-

Received June 20, 2011; revised July 20, 2011; accepted July 24, 2011.

Author contributions: A.M.P. and R.Y. designed research; A.M.P. performed research; A.M.P. analyzed data; A.M.P. and R.Y. wrote the paper.

This work was supported by the Kavli Institute for Brain Science, the National Eye Institute, and the NINDS. We thankE. Fino and V. Nikolenko for inspiration and assistance; L. McGarry, M. Atiya, and Y. Shin for anatomical reconstructions; A. Woodruff, T. Sippy, and M. Dar for help with mice; and members of the laboratory for help and comments.

This article is freely available online through the J Neurosci Open Choice option.

Correspondence should be addressed to Dr. Adam M. Packer, Department of Biological Sciences, Columbia University, 550 West 120th Street, Box 4822, New York, NY 10027. E-mail: adampacker@gmail.com.

DOI:10.1523/JNEUROSCI.3131-11.2011

Copyright $\odot 2011$ the authors $\quad 0270-6474 / 11 / 3113260-12 \$ 15.00 / 0$ niques have been developed to reveal synaptically connected neurons, including paired whole-cell recordings (Thomson and Lamy, 2007), serial EM reconstructions (Denk and Horstmann, 2004; Bock et al., 2011), viral tracing (Wickersham et al., 2007), calcium imaging probing (Peterlin et al., 2000), reverse correlation imaging (Aaron and Yuste, 2006), and photostimulation with caged glutamate (Callaway and Katz, 1993). We recently introduced a variant of photostimulation that uses two-photon uncaging of glutamate to map connected neurons with single-cell resolution (Nikolenko et al., 2007). When combined with uncaging of RuBi-Glutamate, a recently developed caged glutamate compound (Fino et al., 2009), two-photon photostimulation can be applied to map inhibitory connections. Indeed, using RuBi-Glutamate, we recently mapped the synaptic circuits of somatostatin-positive interneurons in the upper layers of mouse frontal cortex, finding a very dense and unspecific connectivity from these interneurons to neighboring pyramidal cells (Fino and Yuste, 2011). This dense connectivity has not been reported before and we wondered whether it was a peculiar feature of the somatostatin-positive interneurons or of the frontal cortex.

To answer this question, we mapped the connectivity from fast-spiking parvalbumin-positive $(\mathrm{PV}+)$ basket cells-the largest population of interneurons in the neocortex-to pyramidal cells (PCs) in different cortical layers and areas. We found locally dense and unspecific connections from PV + interneurons to PCs in two different cortical areas (somatosensory and frontal) as well as in two different layers (2/3 and 5). Although we used brain slices, in which many connections are severed, we often found that every local PV + cell was connected to every PC sampled. Our results support the view that the dense architecture of interneuron connectivity, whereby a "blanket of inhibition" is extended on local pyramidal cells, is a universal or canonical feature in the design of neocortical microcircuits. 


\section{Materials and Methods}

Slice preparation and electrophysiology. All animal handling and experimentation was done according to the National Institutes of Health and local Institutional Animal Care and Use Committee guidelines. Somatosensory or frontal coronal slices $350 \mu \mathrm{m}$ thick were prepared from P12-

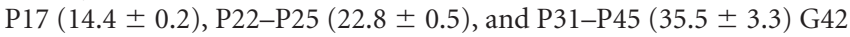
mice of either sex (Chattopadhyaya et al., 2004) using a Leica VT1000S or VT1200S vibratome with ice-cold sucrose solution containing the following (in mM): $27 \mathrm{NaHCO}_{3}, 1.5 \mathrm{NaH}_{2} \mathrm{PO}_{4}, 222$ sucrose, $2.5 \mathrm{KCl}, 3$ $\mathrm{MgSO}_{4}$, and $1 \mathrm{CaCl}_{2}$. Slices were incubated at $36^{\circ} \mathrm{C}$ for $30 \mathrm{~min}$ in ACSF containing the following (in mM): $126 \mathrm{NaCl}, 26 \mathrm{NaHCO}_{3}, 1.1 \mathrm{NaH}_{2} \mathrm{PO}_{4}$, 10 glucose, $3 \mathrm{KCl}, 3 \mathrm{MgSO}_{4}$, and $1 \mathrm{CaCl}_{2}$. During recordings made at $32^{\circ} \mathrm{C}$, ACSF was similar except for the following (in mM): $2 \mathrm{MgSO}_{4}$ and 2 $\mathrm{CaCl}_{2}$. All sucrose and ACSF solutions were saturated with $95 \% \mathrm{O}_{2}$ and $5 \% \mathrm{CO}_{2}$.

Whole-cell recordings were made using the Multiclamp 700B amplifier (Molecular Devices), digitized with National Instruments 6259 multichannel cards, and recorded using custom software written using the LabView platform (National Instruments). Current-clamp recordings were performed with intracellular solution, $\mathrm{pH} 7.2$, containing the following (in mM): $135 \mathrm{~K}$-methylsulfate, $8 \mathrm{NaCl}$, 10 HEPES, $2 \mathrm{Mg}$-ATP, 0.3 Na-GTP, 7 phosphocreatine, 0.02 Alexa Fluor 594, and 10.7 biocytin. Voltage-clamp recordings were performed with intracellular solution, $\mathrm{pH} 7.3$, containing the following (in $\mathrm{mm}$ ): $128 \mathrm{CH}_{3} \mathrm{CsO}_{3} \mathrm{~S}, 10$ HEPES, 10 Na-phosphocreatine, $2 \mathrm{MgCl}_{2}, 3 \mathrm{MgSO}_{4}, 4 \mathrm{Mg}$-ATP, $0.4 \mathrm{Na}$-GTP, 0.02 Alexa Fluor 594, and 10.7 biocytin.

Imaging and RuBi-Glutamate uncaging. Imaging and uncaging were performed as previously described (Fino and Yuste, 2011). Briefly, images were acquired using a custom-made two-photon laser scanning microscope based on the Olympus FV-200 system (side-mounted to a BX50WI microscope with a $40 \times 0.8 \mathrm{NA}$ or $20 \times 0.5 \mathrm{NA}$ water-immersion objective) and a Ti:sapphire laser (Chameleon Ultra II, $>3 \mathrm{~W}, 140 \mathrm{fs}$ pulses, $80 \mathrm{MHz}$ repetition rate; Coherent). Images were acquired at 850 or $900 \mathrm{~nm}$ for GFP and $800 \mathrm{~nm}$ for Alexa 594 with minimal power to avoid uncaging RuBi-Glutamate.

RuBi-Glutamate (300 $\mu \mathrm{M}$; Tocris Bioscience) was added to the oxygenated ACSF during mapping experiments and recirculated with a peristaltic pump (RP-1; Rainin Instruments). All mapping experiments were conducted using the $20 \times 0.5 \mathrm{NA}$ objective.

We used custom software (Nikolenko et al., 2007) to create the complex targets around cell bodies shown in Figure 1D. Each complex target, which consisted of five beamlets due to the diffractive optical element we used (Fig. 1H,I) (Nikolenko et al., 2007), was illuminated for $8 \mathrm{~ms}$, resulting in a stimulation duration of $72 \mathrm{~ms}$ given an additional millisecond to move between each target. A Pockels cell (Conoptics) allowed us to control power precisely over these short durations. The power at the sample ranged from 150 to $330 \mathrm{~mW}$ for mapping experiments. We used multiple different power levels in each mapping experiment, always testing the maximum level to be sure not to miss connections from any PV+ interneurons with particularly high rheobases.

Biocytin histochemistry and reconstructions. At the end of an experiment, slices were fixed and kept overnight in $4 \%$ paraformaldehyde in 0.1 M phosphate buffer $(\mathrm{PB})$ at $4^{\circ} \mathrm{C}$. The slices were then rinsed three times for $5 \mathrm{~min}$ per rinse on a shaker in $0.1 \mathrm{M} \mathrm{PB}$. They were placed in $30 \%$ sucrose mixture ( $30 \mathrm{~g}$ of sucrose dissolved in $50 \mathrm{ml}$ of $\mathrm{ddH}_{2} \mathrm{O}$ and $50 \mathrm{ml}$ of $0.24 \mathrm{M} \mathrm{PB}$ ) for $2 \mathrm{~h}$ and then frozen on dry ice in tissue-freezing medium. The slices were kept overnight in a $-80^{\circ} \mathrm{C}$ freezer. The slices were defrosted and the tissue-freezing medium was removed by three $20 \mathrm{~min}$ rinses in $0.1 \mathrm{M} \mathrm{PB}$ while on a shaker. The slices were kept in $1 \%$ hydrogen peroxide in $0.1 \mathrm{M} P B$ for $30 \mathrm{~min}$ on the shaker to pretreat the tissue, then were rinsed twice in $0.02 \mathrm{M}$ potassium phosphate saline (KPBS) for 20 min on the shaker. The slices were then kept overnight on the shaker in avidin-biotin-peroxidase complex. The slices were then rinsed three times in $0.02 \mathrm{M} \mathrm{KPBS}$ for $20 \mathrm{~min}$ each on the shaker. Each slice was then

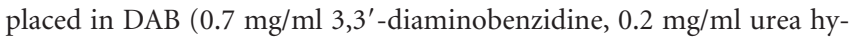
drogen peroxide, $0.06 \mathrm{M}$ Tris buffer in $0.02 \mathrm{M} \mathrm{KPBS}$ ) until the slice turned light brown, then immediately transferred to $0.02 \mathrm{M}$ KPBS, and finally transferred again to fresh $0.02 \mathrm{M}$ KPBS after a few minutes. The stained slices were rinsed a final time in $0.02 \mathrm{M} \mathrm{KPBS}$ for $20 \mathrm{~min}$ on a shaker. Each slice was observed under a light microscope and then mounted onto a slide using crystal mount.

Successfully filled and stained neurons were then reconstructed using Neurolucida software (MicroBrightField). The neurons were viewed with a $100 \times$ oil objective on an Olympus IX71 inverted light microscope or an Olympus BX51 upright light microscope. The Neurolucida program projected the microscope image onto a computer drawing tablet. The neuron's processes were traced manually while the program recorded the coordinates of the tracing to create a digital, threedimensional reconstruction. The $x$ - and $y$-axes formed the horizontal plane of the slice, while the $z$-axis was the depth. The user defined an initial reference point for each tracing. The $z$-coordinate was then determined by adjustment of the focus. In addition to the neuron, the pia and white matter were drawn. Axon and dendrite densities were calculated from the Neurolucida reconstruction using the TREES toolbox (Cuntz et al., 2010). The densities were calculated with voxels $5 \mu \mathrm{m}$ on each side.

Data analysis. Off-line analysis was conducted using MATLAB (Mathworks), InStat (GraphPad), MiniAnalysis (Synaptosoft), and Oriana (Kovach Computing Services). Additional circular statistics tests were performed with the MATLAB CircStat Toolbox (Berens, 2009). All results are expressed as mean \pm SEM.

Monosynaptic connections were characterized by evoking singleaction potentials in the $\mathrm{PV}+$ interneuron and recording the IPSC evoked in the PC to measure the latency, amplitude, rise time, decay time, rate of rise, and failure rate. The rise time is the time elapsed from when the IPSC starts until the peak is reached. The decay time is the time it takes for the membrane potential to return to $37 \%$ of the amplitude of the peak. The rate of rise is the amplitude divided by the rise time. These values were recorded at $+40 \mathrm{mV}$ with a chloride reversal potential of $-80 \mathrm{mV}$ and analyzed with MiniAnalysis. Trains of eight action potentials delivered at $50 \mathrm{~Hz}$ followed by a single action potential $1 \mathrm{~s}$ after the last action potential in the train were evoked in the PV+ interneuron to measure the paired pulse ratio, summation, train depression, and recovery (Markram et al., 1998). Paired pulse ratio is the amplitude of the second IPSC divided by the amplitude of the first IPSC. Summation is the amplitude of the second IPSC relative to the baseline of the first IPSC divided by the amplitude of the first IPSC. Train depression is the amplitude of the last IPSC in the train divided by the amplitude of the first IPSC in the train. Recovery is the amplitude of the IPSC evoked by the action potential $1 \mathrm{~s}$ after the train divided by the amplitude of the first IPSC in the train.

\section{Results}

Two-photon photostimulation of parvalbumin interneurons

To study the connectivity from a well defined subset of interneurons onto nearby PCs, we used the G42 transgenic mouse line in which parvalbumin-expressing interneurons are labeled with GFP (Chattopadhyaya et al., 2004). In coronal brain slices, we targeted and recorded from 91 GFP-positive cells (69 in somatosensory cortex layer $2 / 3,15$ in somatosensory cortex layer 5 , and 7 in frontal cortex layer 2/3), and performed anatomical and electrophysiological characterizations of these neurons. Anatomically, all targeted PV + interneurons had basket cell features with promiscuous and densely branching axons $(n=15$ reconstructions; Fig. 1A). Electrophysiologically, GFP-positive neurons were all fast-spiking cells, firing at frequencies $>50 \mathrm{~Hz}(97 \pm 6$ $\mathrm{Hz}, n=42$ ) (Ascoli et al., 2008). They also had high rheobases $(312 \pm 102 \mathrm{pA}, n=42)$, strong fast afterhyperpolarization (fAHP) currents, and rectifying I/V curves (Fig. $1 B, C$ ) (Woodruff et al., 2009).

To map synaptic connections from PV + interneurons onto postsynaptic PCs, we followed the protocol initially developed by Nikolenko et al. (2007) and recently refined for inhibitory connections by Fino and Yuste (2011). This protocol relies on the ability to optically stimulate individual neurons with two-photon uncaging of RuBi-Glutamate (Fino et al., 2009). We achieved single-cell resolution of photoactivation by multiplexing the two- 
A

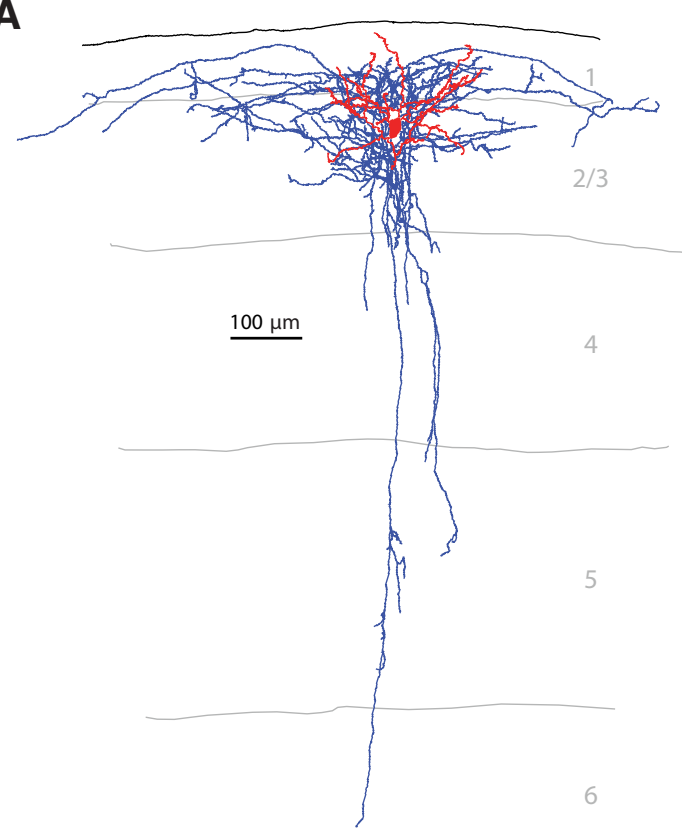

B

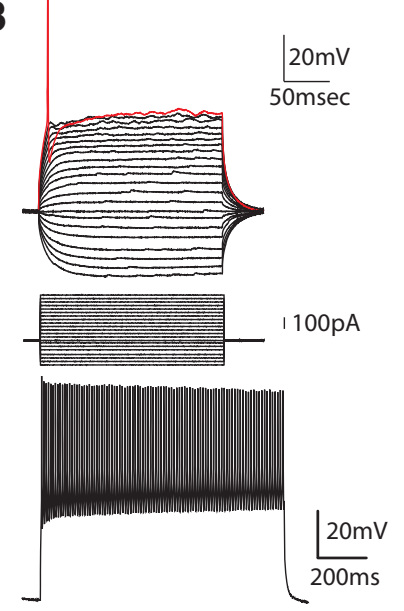

C

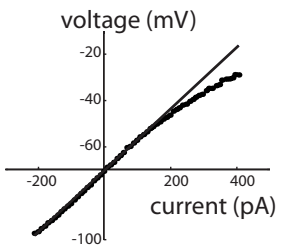

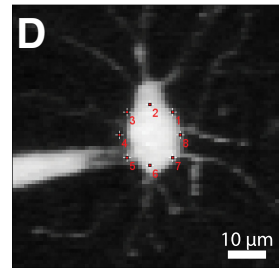

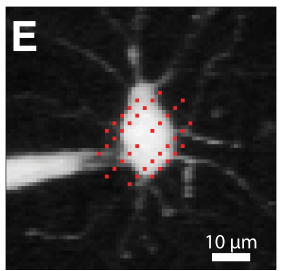

H

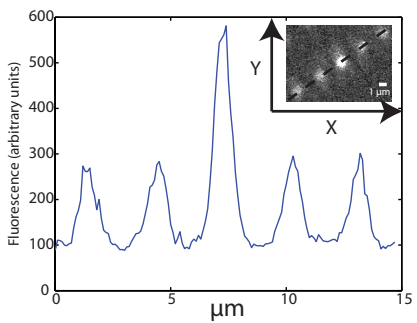

J

000000000000000

000000000000000

000000000000000

000000000000000

000000000000000

$000000^{2} 01000000000$

$000000 \frac{1}{3}=000000000$

000000000000000

000000000000000

000000000000000

$000000000000000_{25}$

$000000000000000 \mu$
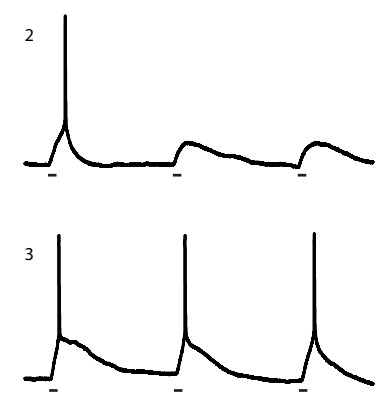

Figure 1. Characterization and photostimulation of parvalbumin-positive fast-spiking basket cells. $\boldsymbol{A}$, Reconstruction of a GFP-positive interneuron in somatosensory cortex layer 2/3 from the G42 transgenic mouse line (axons in blue, dendrites in red). $\boldsymbol{B}$, Electrophysiological recordings revealed the classic high rheobase and fAHP current (top). A fast-spiking response was elicited after injecting the interneuron with $1 \mathrm{nA}$ of current (bottom). C, This subtype of interneuron is characterized by a rectifying IV curve (Woodruff et al., 2009). D, E, A two-photon laser beam was multiplexed across time by sequentially stimulating multiple targets around a cell body $(\boldsymbol{D})$ and across space by engaging a diffractive optical element to split the laser beam into five beamlets $(\boldsymbol{E})$ to photon laser beam across time, focusing it on eight points around the target cell soma (Fig. $1 D-G$ ) and across space by using a diffractive optical element (Nikolenko et al., 2007) to split the laser beam into five beamlets (Fig. 1E). This strategy preserved the integrity of the point-spread function of the two-photon beam (Fig. $1 H, I)$, thus enabling single-cell precision. When photostimulating a recorded $\mathrm{PV}+$ interneuron with this method, one to several action potentials (APs) were elicited at intermediate laser powers $(150 \mathrm{~mW}$ on sample; Fig. $1 F$ ) while bursts of APs were elicited at greater powers $(300 \mathrm{~mW}$ on sample; Fig. $1 G$ ). Despite the high rheobase of these cells, these laser powers $(>150 \mathrm{~mW})$ never failed to produce APs (APs in 21 of 21 cells tested). APs induced by uncaging displayed the AAHP waveform and high-frequency trains characteristic of fast-spiking cells (Fig. 1G). In addition, when the stimulation of the $\mathrm{PV}+$ interneuron was repeated every $5 \mathrm{~s}$, identical responses were produced each time (14.22 $\pm 0.08 \mathrm{~ms}$ delay to first spike, $11.6 \pm 0.2$ action potentials per stimulation, $n=10$ photostimulations). Thus, the photostimulation strategy we used was precise and reliable, activating neurons with single-cell resolution.

To test whether our photostimulation inadvertently activated nearby neurons, we patched PCs with a low rheobase (40 $\mathrm{pA}$ ) and photostimulated the entire area surrounding the cell in a grid fashion using the same parameters as we did to stimulate interneurons. First, we confirmed that this PC could be photostimulated by eliciting action potentials via uncaging

uncage RuBi-Glutamate around a cell body (laser spots are drawn at a(tual size, see $\boldsymbol{H}-\boldsymbol{I})$. $\boldsymbol{F}$, Single action potentials were elicited via illumination with $150 \mathrm{~mW}$ of $800 \mathrm{~nm}$ light on the sample using a $20 \times 0.5$ NA objective (black bar shows duration of illumination). G, At a higher power ( $300 \mathrm{~mW}$ ) multiple action potentials were elicited with the characteristic fast-spiking waveform. $\boldsymbol{H}$, The point-spread function of the diffractive optical element beamlets was measured with the $20 \times 0.5 \mathrm{NA}$ objective we used for mapping and $0.17 \mu \mathrm{m}$ fluorescent beads. The lateral size of each diffractive optical element beamlet is $\sim 1 \mu \mathrm{m}$ (inset, raw image). $I$, The axial resolution of each beamlet is $6 \mu \mathrm{m}$. $J$, Direct test of two-photon photostimulation spatial resolution. The locations of 180 stimulation targets that were stimulated during the recording of the $\mathrm{PC}$ (center, black) are indicated by black circles. Location 1, The target indicated in blue, directly on the soma of the PC, was photostimulated directly to produce bursts of action potentials 10 times (one representative example is shown at right, top). Location 2, This target (upper red circle), which was just slightly offset from the center of the patched PC, caused the cell to fire only once (right, middle). Location 3, Photostimulation of this target (lower red circle) also caused the patched PC to fire single action potentials (right, bottom). 
over the soma directly 10 of 10 times (Fig. $1 \mathrm{~J}$, top right trace). Next, we photostimulated, with the same laser power, a grid of 180 locations in the field of view (Fig. $1 J$ ) three times each and found that the PC recorded from only produced action potentials when two locations where stimulated: one just slightly offset from the soma (Fig. $1 \mathrm{~J}$, location 2, red circle) and one on the bottom of the soma (Fig. 1J, location 3, red circle). We concluded that it is very rare that action potentials are elicited unless stimulating a cell soma directly. This makes it unlikely that nontargeted neurons could be led to produce action potentials by inadvertent photostimulation, especially for interneurons that have higher rheobases, again confirming the high resolution of this technique.

\section{Mapping monosynaptic connections from $\mathrm{PV}+$ interneurons to pyramidal cells}

We then mapped inputs from interneurons onto PCs, following the strategy developed by Fino and Yuste (2011). Briefly, a PC was patched while nearby $\mathrm{PV}+$ interneurons were sequentially photostimulated (Fig. $2 \mathrm{~A}$, blue arrow). Each PV+ interneuron was activated with a range of at least five laser powers, including the maximum of $300 \mathrm{~mW}$ on the sample. Postsynaptic responses in the PC that were time-locked to the stimulus were measured in voltage clamp (Fig. 2B, cells 1-5). The PC was initially held at $+40 \mathrm{mV}$ to increase the driving force on inhibitory currents and then at $-40 \mathrm{mV}$ to test that these postsynaptic currents were indeed inhibitory (Fig. 2B, lower trace).

If the photostimulation of a PV + cell generated a synaptic response in the PC with a GABAergic reversal potential (set to $-80 \mathrm{mV}$ ), we identified them as true positive, i.e., a putatively connected neuron, since it was time-locked to the optical stimulation of an inhibitory cell (Fig. $2 B$, red box). To test whether interneurons generating these true positive responses were indeed connected to the postsynaptic PC cell, we patched them with a second electrode (Fig. $2 A-D$, cell 4 , red arrows), stimulated them with intracellular pulses of current to produce action potentials, and confirmed the generation of IPSCs (Fig. 2E). Overall, we found that, in nine of 10 cases, true positive PV + cells generated short latencies IPSCs in the PC, indicative of monosynaptic transmission $(1.12 \pm 0.19 \mathrm{~ms}, n=9$ pairs; Fig. $2 G-J)$. These presynaptic $\mathrm{PV}+$ interneurons always fired APs when photostimulated (Fig. 1G) and generated inhibitory responses with large outward currents (Fig. 2D, $H$ ).

To explore whether interneurons that did not generate a response in the PC could be potentially also connected to it, we also patched eight photostimulated PV+ interneurons for which no response was observed (negatives). We confirmed that there was no synaptic connection in any of these cases (Fig. $2 \mathrm{~K}-\mathrm{N}$ ), concluding that negatives were true negatives. In other words, we never observed any false negatives in which a PV+ interneuron that did not appear to be connected optically was in fact connected. Even PV + interneurons that were very close to each other could be disambiguated, as evidenced by the complete lack of response (Fig. $2 B$, cell $5 ; F$ ) from cells directly adjacent to true positive connected cells (Fig. $2 \mathrm{~A}$, gray arrow vs red arrow).

In addition to true positive and negative responses, we occasionally observed unexpected postsynaptic currents that reversed direction at the glutamate reversal potential of $0 \mathrm{mV}$ (false positives responses). Most of these false positives occurred when directly stimulating in the vicinity of the recorded PC, just outside its soma (Fig. 2O). In addition to a different reversal potential, these responses had slower kinetics (Fig. $2 P$ ) than the true positive responses from connected PV+ interneurons (Fig. $2 \mathrm{H}$ ). We also observed occasional false positive responses that arose from stimulating sites far away from the dendritic tree of the recorded neuron (Fig. 2Q, black circle). We presume that these excitatory responses (Fig. $2 R$ ) are due to PCs located nearby to the targeted $\mathrm{PV}+$ interneuron, which were accidentally stimulated, consistent with our grid control stimulation (Fig. $1 \mathrm{~J}$ ). These two types of false positive responses were rare and easily distinguished from true positive responses because of their glutamatergic reversal potentials.

In summary, we classified all stimulated interneurons into three types, based on their responses in the PC cell: (1) true positive cells, connected to the PC with monosynaptic IPSCs; (2) true negative cells, not connected; and (3) false positive cells, which generated contaminating EPSCs. For the remainder of the study, we used this classification to analyze the maps of input connectivity.

\section{Dense local connectivity from PV+ interneurons onto pyramidal cells in different cortical layers and areas}

We assembled a total of 82 input maps from layers $2 / 3(n=38)$ and $5(n=21)$ of somatosensory cortex and layer $2 / 3$ of frontal cortex $(n=23)$, arising from recordings from 13 single PCs, 12 PC pairs, 11 PC triplets, and three PC quadruplets. In optimal cases, we could map the inputs onto four PCs in different focal planes (Fig. 3) For example, in the experiment shown, we tested the connectivity from $22 \mathrm{PV}+$ interneurons, assessing the connectivity status of a total of 88 pairs in this single slice. This approach allowed for large-scale measurement of connectivity within one slice whereas past dual whole-cell recording that maintained single-cell accuracy necessitated comparisons across many different slices.

Using this three-dimensional strategy with simultaneous whole-cell recordings from multiple PCs, we were able to sample the connectivity from $2002 \mathrm{PV}+$ interneuron to PC pairs. On average, $24 \pm 1 \mathrm{PV}+$ interneurons were tested for each map from $5 \pm 0.2$ focal planes, and we detected connections at all depths examined. The correlation between the connection probability and the depth of the patched PC was significant but small $\left(R^{2}=0.08, p<0.05\right.$ ANOVA, $n=82$ maps, data not shown).

We found that PCs received connections from most local $\mathrm{PV}+$ interneurons but not from more distantly located ones (Figs. 3, 4A). Overall, we tested a total of $21 \pm 1,29 \pm 3$, and $25 \pm$ $2 \mathrm{PV}+$ interneurons per $\mathrm{PC}$ in $\mathrm{S} 2 / 3, \mathrm{~S} 5$, and $\mathrm{F} 2 / 3$, respectively ( $p>0.05$, Kruskal-Wallis), observing an average of $3 \pm 0.4,10 \pm$ 1.4 , and $7 \pm 0.8$ connections in S2/3, S5, and F2/3 input maps, respectively. These differences in connections between cortical areas and layers were statistically significant (one-way ANOVA, $p<0.0001$; Tukey multiple-comparison test, $\mathrm{S} 2 / 3$ vs $\mathrm{S} 5, p<$ $0.001 ; \mathrm{S} 2 / 3$ vs F2/3, $p<0.01$; S5 vs F2/3, $p<0.05$; Fig. $4 B$ ).

To analyze the dependence of the connectivity versus intersomatic distance, we defined the connection probability from $\mathrm{PV}+$ interneurons to a PC as a percentage (connected interneurons divided by connected and unconnected interneurons) and measured it as a function of intersomatic distance between interneurons and PCs. We assembled input maps from rectangular slabs of cortex measuring 800 by 600 by $52 \pm 3 \mu \mathrm{m}$ (Fig. $4 C$ ) so we were able to calculate intersomatic distance in three dimensions (Fig. $4 D$, green bars). False positives were rare and excluded from subsequent analyses (Fig. $4 D$, black bars).

In all areas, the probability of connection was remarkably high in the local range and inversely proportional to intersomatic distance between the PV+ interneuron and the PC (Fig. 4E). An 

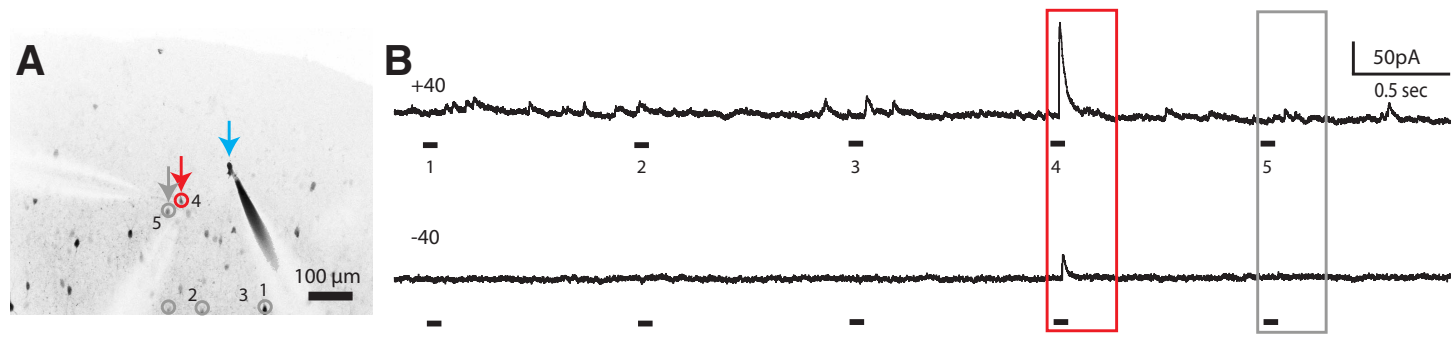

C

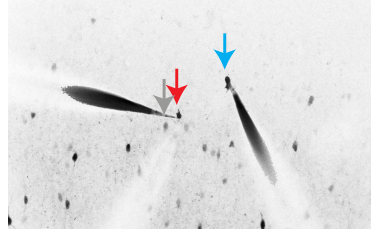

D

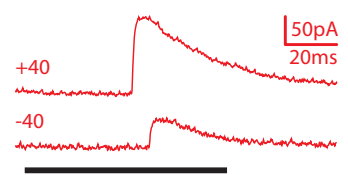

E

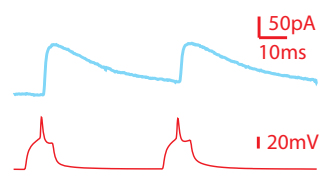

$\mathbf{F}$

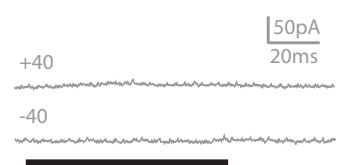

Optically detected inhibitory connection

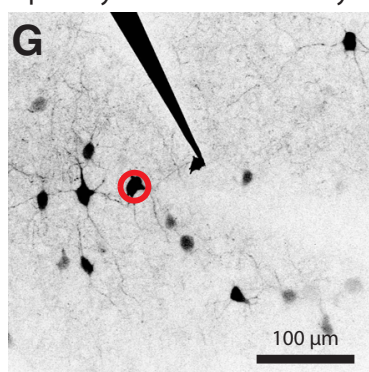

Optically detected no connections

K
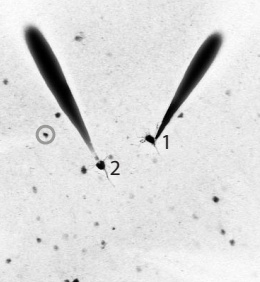

$\cdots$

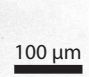

False positive - direct stimulation
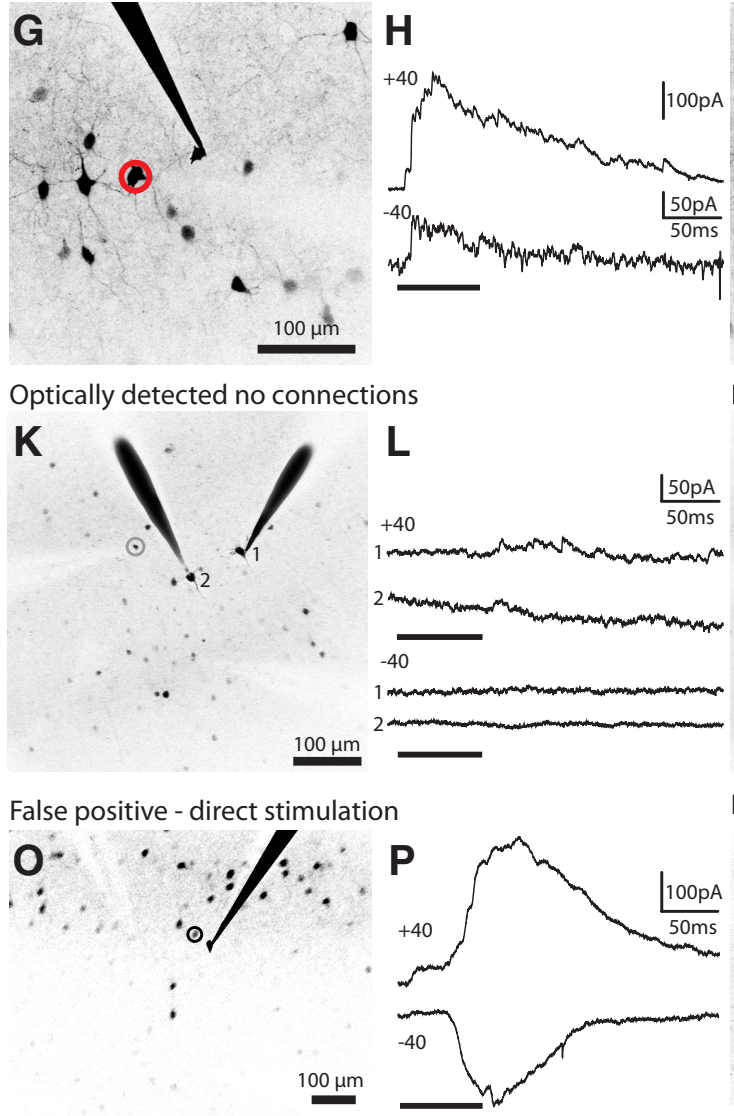

Connection confirmation

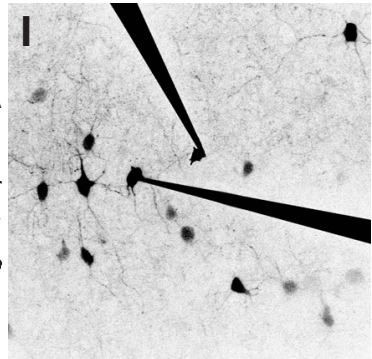

J

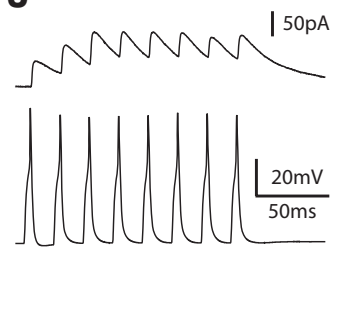

No connections confirmation
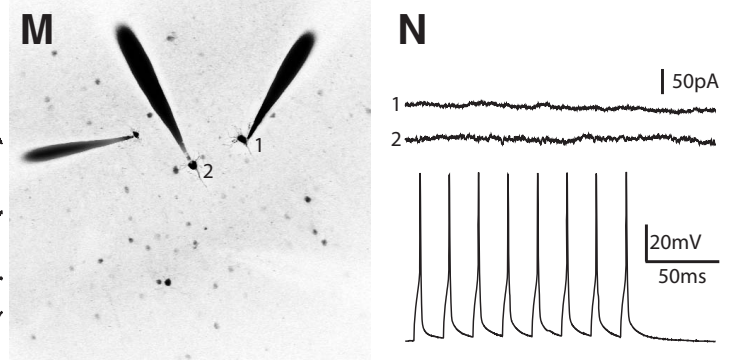

False positive - excitatory connection
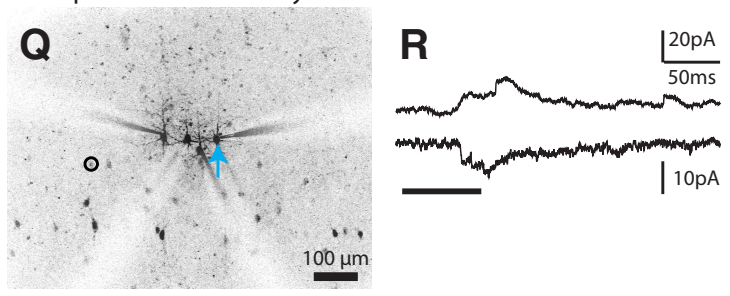

Figure 2. Mapping inhibitory inputs of pyramidal cells. A, Mapping potential connections from five interneurons (numbered 1 to 5 ) to one $P C$ (blue arrow) in somatosensory layer 2/3. Gray circles, Unconnected interneurons; red circles, connected. $\boldsymbol{B}$, Electrophysiological recordings obtained from the $\mathrm{P}$ (at holding potentials of $+40 \mathrm{mV}$ (top) and $-40 \mathrm{mV}$ (bottom) during the photostimulation of the interneurons shown in $\boldsymbol{A}$. Note the synaptic response from one interneuron $(\boldsymbol{A}$, red arrow; higher magnification in $\boldsymbol{D})$ while an interneuron directly nearby $(\boldsymbol{A}$, gray arrow; higher magnification in $\boldsymbol{F}$ ) showed no response. $\boldsymbol{C}, \boldsymbol{E}$, The red PV + interneuron neuron was patched and confirmed to be synaptically connected while no response was recorded for the gray neuron $(\boldsymbol{F})$, which was determined to be unconnected by photostimulation. G, True positive response. A PC patched in a field of view showing many GFP-positive PV+ interneurons nearby, one of which (red circle) was determined to be putatively connected by optical stimulation. $\boldsymbol{H}$, While holding the PC at $+40 \mathrm{mV}$ (top) or $-40 \mathrm{mV}$ (bottom), we photostimulated the cell circled in $\mathbf{G}$, eliciting IPSCs. The reversal potential for GABA was set at $-80 \mathrm{mV}$ in the internal solution so that EPSCs, but not IPSCs, would change directions between +40 and $-40 \mathrm{mV}$. I, J, The photostimulated cell was patched, confirming electrophysiologically that it was indeed synaptically connected. $\boldsymbol{K}$, Negative responses. A PV + interneuron (gray circle) was targeted for photostimulation during whole-cell recording of two nearby PCs. $L$, The recordings from the PCs in $\boldsymbol{K}$ show no evidence of a synaptic connection during photostimulation of the PV + interneuron. $\boldsymbol{M}, \boldsymbol{N}$, The photostimulated cell was patched, confirming electrophysiologically the lack of synaptic connections from the PV + interneuron onto either PC. $\mathbf{0}$, False positive response. APV + interneuron (black circle) directly next to the recorded $\mathrm{PC}$ was targeted for photostimulation. $\boldsymbol{P}$, A slow response, which was outward at $+40 \mathrm{mV}$ but flipped at $-40 \mathrm{mV}$, was distinguished from the synaptic event shown in $\boldsymbol{H}$ as a direct stimulation of the patched neuron. Q, A PV + interneuron (black circle), located distal to the recorded PC (blue arrow), was targeted for photostimulation. $\boldsymbol{R}$, An excitatory cell connected to the recorded PC was stimulated, resulting in a false positive distinguished by the presence of EPSCS. 

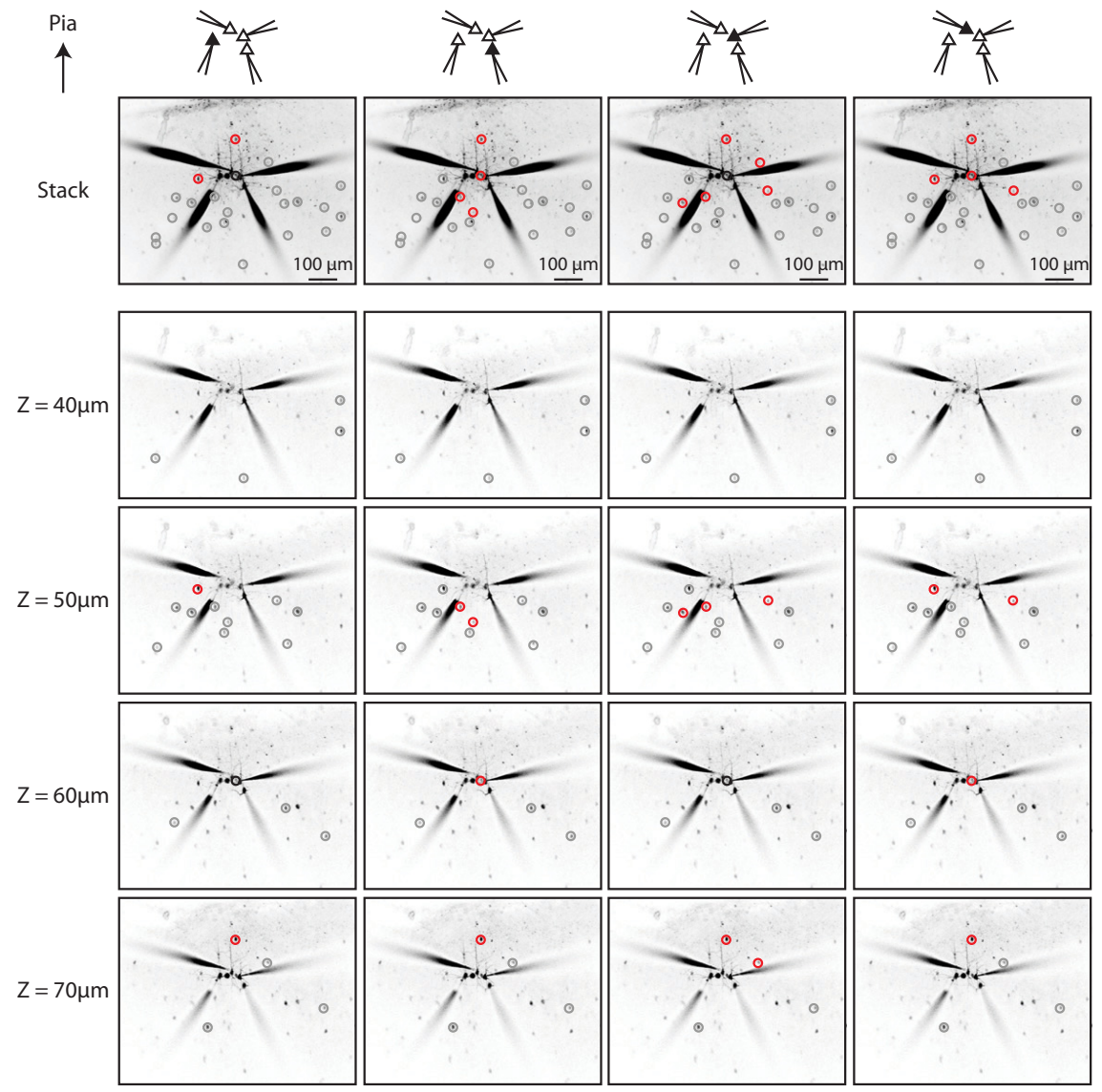

Figure 3. Simultaneous mapping of PV + inputs to four PCs. Four PCs in somatosensory cortex layer $2 / 3$ were patched and the surrounding PV + interneurons in four different focal planes were optically stimulated. Each column shows the result of mapping the GFP-labeled PV + interneurons for each of the four PCs. Each row shows the result from a different focal plane, with the projection of all the focal planes in the top row. Connected PV + interneurons are circled in red, unconnected PV + interneurons are circled in gray, and PV + interneurons at which a direct stimulation of the patched $\mathrm{PC}$ occurred are circled in black.

exponential fit of the connection probability versus intersomatic distance revealed spatial decay constants of 124, 183, and $180 \mu \mathrm{m}$ for S2/3, S5, and F2/3, respectively $\left(R^{2}=0.92\right.$, 0.91 , and 0.86 , respectively).

For intersomatic distances of $<200 \mu \mathrm{m}$, the probability of connection from a $\mathrm{PV}+$ interneuron to a $\mathrm{PC}$ was high, ranging from $43 \pm 6 \%$ in somatosensory layer $2 / 3$, to $67 \pm 6 \%$ in somatosensory layer 5 , and up to $76 \pm 4 \%$ in frontal layer $2 / 3$ (one-way ANOVA, $p<0.0001$; Tukey multiple-comparisons posttest, S2/3 vs S5, $p<0.05$; S2/3 vs F2/3, $p<0.001$; 55 vs F2/3, $p>0.05 ; n=$ 38,20 , and 23 maps in S2/3, S5, and F2/3, respectively; Fig. $4 F$ ). In fact, at these close distances we found 13 (of 82) cases of completely connected local maps $(n=5$ for S2/3; $n=4$ for S5, $n=4$ for $\mathrm{F} 2 / 3$; ranging from 1 to 7 interneurons, average $3.4 \pm 0.6$ interneurons, $n=13$ maps).

Taking into account all distances, the average connection probability (connected out of connected and unconnected neurons) was lower in S2/3 (18 $\pm 3 \%)$ than in S5 (36 $\pm 4 \%)$ or F $2 / 3$ $(33 \pm 3 \%)(p=0.001$, Kruskal-Wallis; $p<0.01$ for S2/3 vs S5 and $\mathrm{S} 2 / 3$ vs F2/3; $p>0.05$ for S5 vs F2/3, Dunn's multiplecomparison test; Fig. $4 G$ ). A similar result was found when comparing the histograms of connectivity versus distance (Fig. $4 E$ ) in a bin-by-bin fashion ( $p<0.0001$, Friedman test). This difference was not due to a difference in the number of targets tested in the different areas and layers, variables that were not significantly different (Fig. 4B).
We also acquired $12 \mathrm{PV}+$ input maps for PCs in S2/3 in mature animals $(n=8$ P22-P25, $n=4$ P31-P45; Fig. $4 H, I)$. The connection probability within $200 \mu \mathrm{m}$ was consistently high at all ages $(43 \pm 6 \%$ $\mathrm{P} 13-\mathrm{P} 15,41 \pm 9 \% \mathrm{P} 22-\mathrm{P} 25,48 \pm 19 \%$ P31-P45; $p>0.05$, Kruskal-Wallis). Taking into account all distances, the average connection probability appeared to decrease; this effect was not statistically significant $(18 \pm 3 \%$ P13-P15, $13 \pm 2 \%$ P22-P25, $9 \pm 2 \%$ P31-P45; $p>0.05$, Kruskal-Wallis). Thus, we found no strong evidence for pruning of interneuron to PC connections as the locally dense architecture remains intact in mature animals.

\section{Confirmation of dense local connectivity with dual whole-cell recordings}

The previous results indicated that $\mathrm{PV}+$ interneurons can be very densely connected to their local PCs, and that in some cases this dense connectivity can exist at the physical limit, where every $\mathrm{PV}+$ interneuron is connected to every PC sampled.

Given that this result was obtained with a new mapping method, we sought to confirm the high local connectivity with dual whole-cell recordings in nonoptical experiments by recording from randomly selected pairs of PV + interneurons and PCs whose cell bodies were within $160 \mu \mathrm{m}$ each other (Fig. 5A). These control experiments were performed in animals ages P13-P16. In 27 of 33 pairs recorded in $\mathrm{S} 2 / 3$, the $\mathrm{PV}+$ interneuron was connected to the PC (Fig. $5 B-D$ ). These monosynaptic connections had low failure rates and large amplitudes that depressed over the course of an eight action potential train delivered at $50 \mathrm{~Hz}$, but recovered $1 \mathrm{~s}$ later (Fig. $5 \mathrm{~B}$; Table 1). Restricting the analysis to intersomatic distances of $<100 \mu \mathrm{m}$ showed that 24 of 27 pairs were synaptically connected.

We also confirmed the dense local connectivity in S5 and F2/3 with dual patch-clamp recordings that revealed $86 \%$ (6 of 7) of pairs in S5 and 80\% (4 of 5) of pairs in F2/3 were connected. In two instances in S5, one PV+ interneuron was connected to two nearby PCs (at distances of 60 and $84 \mu \mathrm{m}$ in one experiment and 77 and $92 \mu \mathrm{m}$ in the other).

These dual whole-cell recordings confirmed the locally dense connectivity of PV + interneurons to PCs observed with optical stimulation. For the optical mapping, we observed high probabilities of connection within $100 \mu \mathrm{m}$ from the PC: $71 \pm 9 \%$ in S $2 / 3$ ( $n=21$ maps), $92 \pm 8 \%$ in S5 ( $n=12$ maps), and $80 \pm 7 \%$ in F2/3 ( $n=14$ maps) (Fig. $5 E$ ). Results obtained from optical mapping and patch-clamp recordings were not significantly different $(\mathrm{S} 2 / 3, p=0.28$; S5, $p=0.38$; F2/3, $p=0.13$; MannWhitney; Fig. $5 E$ ). The probability of connection within $100 \mu \mathrm{m}$ also did not differ between areas and layers $(p=0.15$ for mapping, $p=0.86$ for patching, Kruskal-Wallis). This implies that the locally dense inhibitory connectivity is similar across the cortical areas and layers tested. 


\section{Lack of specificity in} interneuron-pyramidal cell connectivity A corollary of locally dense connectivity from PV+ interneurons to PCs is that these interneurons must contact all, or most, PCs nearby, regardless of their identity or adherence to a particular subcircuit. We tested this prediction by comparing the input maps, obtained at the same time, of several PCs from the same slice (Figs. 3, 6). We used these simultaneously acquired maps to examine whether pairs of PCs that were connected among themselves had a preferential innervation from nearby interneurons compared with pairs of PCs that were unconnected between themselves (Fig. 6A) (Yoshimura et al., 2005). This analysis was performed with maps of animals ages P12-P17.

We found that the common connection probability (the number of interneurons connecting to two PCs divided by the number of interneurons connecting to either PC) for pairs of PCs was similar, regardless of whether these PC pairs were connected or not $(0.34 \pm 0.09$ and $0.28 \pm$ 0.07 for connected and unconnected PC pairs, respectively; $p=0.33, n=8$ connected PCs, $n=26$ unconnected PCs, S2/3, Mann-Whitney; Fig. 6B). The common connection probability for all pairs of PCs, connected or not, fell as the distance between PCs increased $(p<0.05$, $n=34$ S2/3 PC pairs; Fig. $6 D$ ). Given that connected pairs often occur at close distances, we were concerned that the common connection probabilities for connected PCs could be biased if the distances between those PCs were systematically smaller. In fact, the average distance of connected PCs was significantly closer than that for unconnected PCs ( $44 \pm 5 \mu \mathrm{m}$ vs $65 \pm 6 \mu \mathrm{m}, p<$ 0.05 , unpaired $t$ test with Welch correction). To avoid this distance effect, we restricted our analysis to PC pairs within $70 \mu \mathrm{m}$ of each other. This reduced the average distance of unconnected PCs to $45 \pm 5 \mu \mathrm{m}$, which was not significantly different from the average distance of connected PCs ( $p=$ $0.9284, n=8$ connected PCs, $n=16$ unconnected PCs, MannWhitney). In this reduced dataset, the common connection probability for unconnected or connected PCs was statistically indistinguishable $(0.35 \pm 0.11$ for unconnected PCs vs $0.34 \pm$ 0.09 for connected PCs, $p=0.7286$, Mann-Whitney). This analysis indicated that $\mathrm{PV}+$ interneurons do not discriminate between PCs that are or are not forming subcircuits.

\section{Spatial patterns of connected PV+ interneurons vary between layers}

The previous analysis of connectivity was performed by taking into account the distance of the interneurons to the pyramidal cell. To further examine the spatial structure of connections for
B
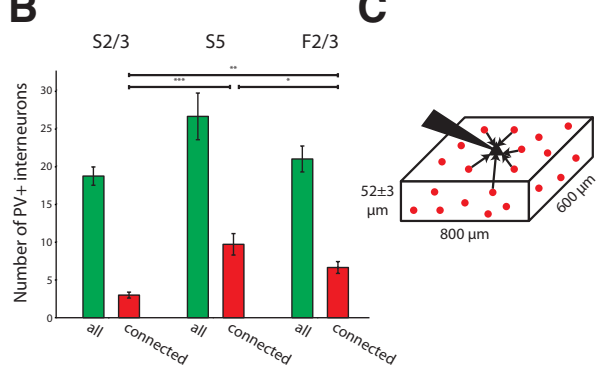

$\therefore 100 \mu \mathrm{m}$
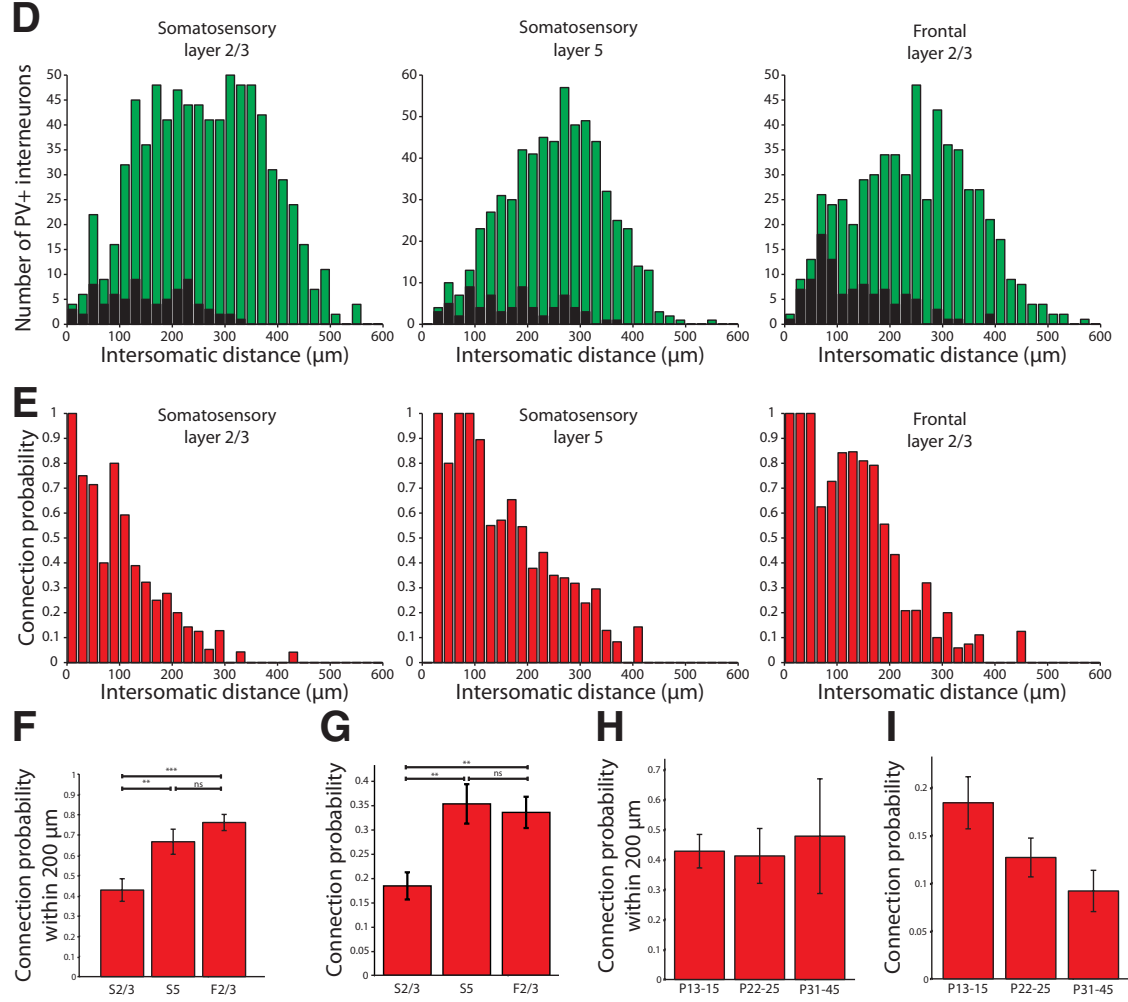

Figure 4. Dense $P V+$ interneuron inputs to $P C s . A$, Representative map from $S 2 / 3$ showing inputs from $P V+$ interneurons to PCs (red, connected; gray, unconnected; black, false positive). $\boldsymbol{B}$, Number of PV + interneurons tested optically per patched PC (no we sampled in our maps. D, Histogram showing all the intersomatic distances of the PV + interneurons from the PCs whose nnectivity was tested (green bars) in the different cortical layers and areas. False positive responses (black bars) were few in number. $\boldsymbol{E}$ of PV + interneurons to $P C$ sin all areas and layers was very high when the $P V+$ interneurons were close to the $P C$, and fell off with distance. in somatosensory layer 5 or frontal layer $2 / 3$. $\boldsymbol{H}$, The probability of observing a connection from a PV + interneuron to a PC within $200 \mu \mathrm{m}$ in older animals (no statistical differences). I, Connection probability for all optically stimulated interneurons in older animals (no statistical differences). n.S., Not significant. ${ }^{*} p<0.05,{ }^{* *} p<0.01,{ }^{* * *} p<0.001$.

the three cortical locations examined, we plotted the position of connected and unconnected PV + interneurons within the coordinate plane of the cortical circuit and used these plots to explore whether the angular position of connected PV + interneurons relative to PCs displayed any particular geometry (Fig. 7A). To visualize this angular distribution, we computed polar plots in which the PCs were at the center and a sector was plotted with a radius proportional to the average connection probability of $\mathrm{PV}+$ interneurons from that angular region (Fig. $7 B$ ). At close distances $(0-200 \mu \mathrm{m})$, the average connection probability from any given angle was homogenously high across areas and layers (Fig. $7 B$, top row). At further distances $(200-600 \mu \mathrm{m})$, a spatial pattern appeared, whereby PCs preferentially received connec- 
A

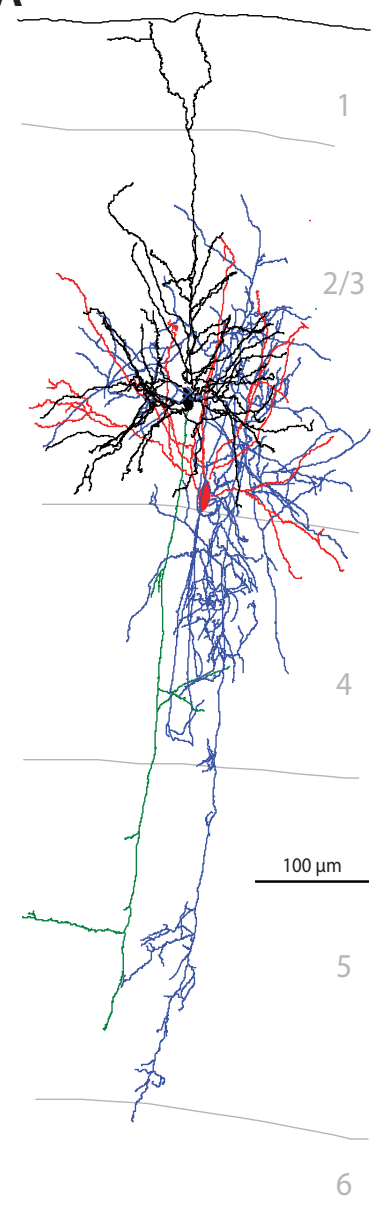

B

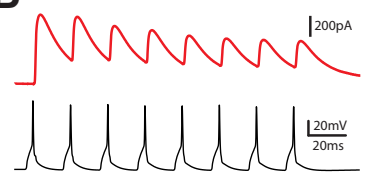

C

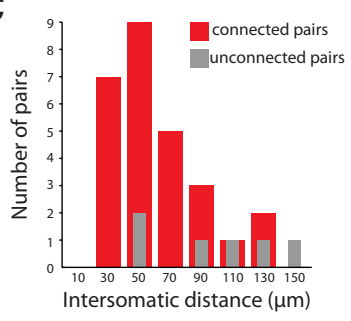

D

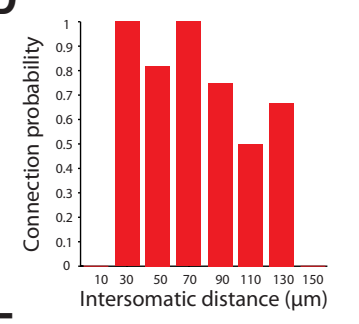

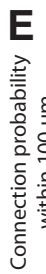

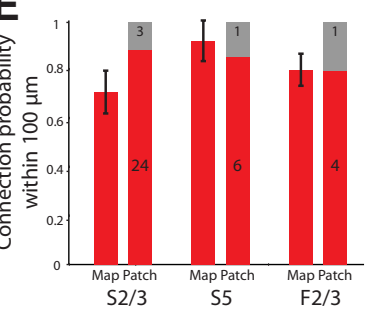

Figure 5. Confirmation of dense PV + innervation of $P C S . A$, Anatomical reconstruction of a connected pair with a PV + interneuron connected to a PC. Blue, PV + axon; red, PV + dendrites; green, PC axon; black, PC dendrite. $\boldsymbol{B}$, Electrophysiological recording of this PV + interneuron to $P C$ connection showing IPSCs in the $P C$ in response to a train of eight action potentials evoked at $50 \mathrm{~Hz}$ in the PV + interneuron. The IPSCs depress over the course of the action potential train. $\boldsymbol{C}$, Intersomatic distances of the tested pairs from dual whole-cell recording experiments (red, connected; gray, unconnected). D, Probability of connection observed at different intersomatic distances. $\boldsymbol{E}$, Connection probability within $100 \mu \mathrm{m}$ did not differ whether observed with mapping or patching in all tested cortical areas and layers. Within this nearby range, there was no difference in connection probability between the layers and areas.

Table 1. Characteristics of the synaptic connections from PV+ interneurons to PCs ( $n=10$ from $\mathrm{S} 2 / 3, \mathrm{S5}$, and F2/3)

\begin{tabular}{lc}
\hline Latency $(\mathrm{ms})$ & $0.87 \pm 0.05$ \\
Amplitude $(\mathrm{pA})$ & $222 \pm 88$ \\
Rise time $(\mathrm{ms})$ & $3.33 \pm 0.42$ \\
Decay time $(\mathrm{ms})$ & $12.21 \pm 1.45$ \\
Rate of rise (pA/ms) & $76.44 \pm 27.66$ \\
Failure rate & $0.10 \pm 0.06$ \\
Paired pulse ratio & $0.91 \pm 0.12$ \\
Summation & $1.34 \pm 0.17$ \\
Train depression & $0.64 \pm 0.11$ \\
Recovery & $0.90 \pm 0.09$ \\
\hline
\end{tabular}

Note that during the recordings, the postsynaptic $\mathrm{PC}$ was held at $+40 \mathrm{mV}$ with the chloride reversal set to $-80 \mathrm{mV}$.

tions from interneurons located in vertically oriented positions (Fig. $7 B$, middle row). Averaging all distances together also revealed a preference for connections from vertical orientations and a difference between layer $2 / 3$ and layer 5 of somatosensory and frontal cortices (Fig. $7 B$, bottom row). While layer $2 / 3$ PCs

A
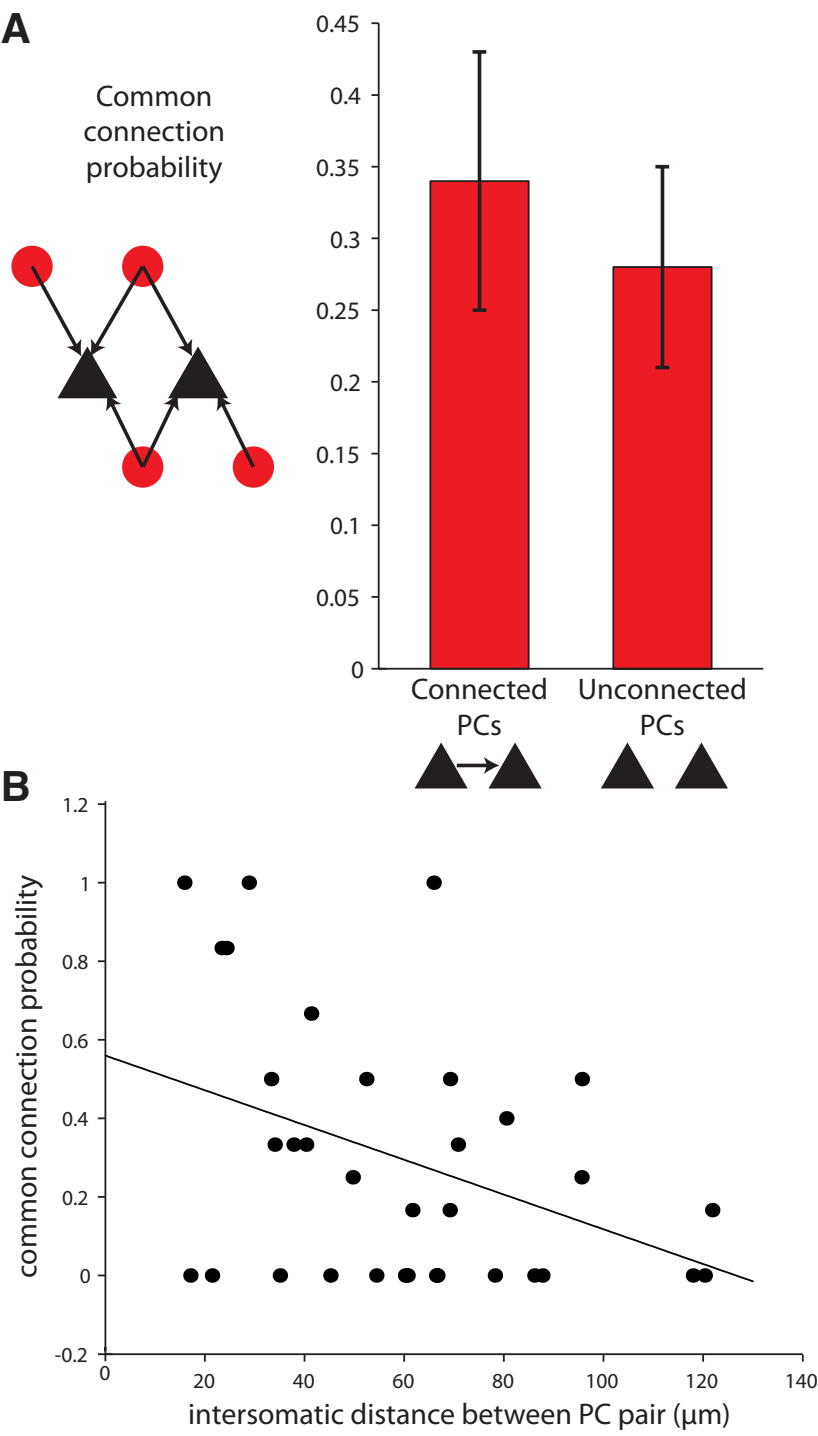

Figure 6. Lack of specificity in PV + interneuron connectivity. $\boldsymbol{A}$, The common connection probability, calculated as the number of PV + interneurons that connect to two PCs out of the number of $P V+$ interneurons that connect to either $P C$, does not differ depending on whether the postsynaptic $\mathrm{PCs}$ are connected to each other. $\boldsymbol{B}$, The common connection probability is inversely proportional to the distance between the PCs ( $r=-0.3898)$.

received connections from locations closer to the pial surface, layer 5 PCs received connections from locations in deeper layers $\left(p=0.0027\right.$, circular ANOVA). Under the convention of $90^{\circ}$ being toward the pial surface, the average direction from which $\mathrm{PCs}$ received connections from $\mathrm{PV}+$ interneurons was $86^{\circ}$ in $\mathrm{S} 2 / 3,258^{\circ}$ in $\mathrm{S} 5$, and $105^{\circ}$ in $\mathrm{F} 2 / 3$; these angles differed significantly $(p<0.01$ between S2/3 and S5, $p<0.01$ between F2/3 and $\mathrm{S} 5, p=0.6494$ between S2/3 and F2/3, Watson-Williams test).

We therefore concluded that there were significant differences in the spatial pattern of connectivity between upper and lower layer PV + cells, although in both cases and both cortical areas, there was a preferential vertical arrangement of presynaptic neurons.

Estimation of convergence and divergence in PV-PCs circuits The high density of connection we observe from PV + interneurons to nearby PCs has two implications: (1) each PC must receive convergent connections from many $\mathrm{PV}+$ interneurons and 
(2) each PV + interneuron must contact many PCs. Using the probability function that describes connectivity, we calculated these numbers and explored whether they are self-consistent. To do this, we constructed a cube of neocortex, $500 \mu \mathrm{m}$ per side, with one PC located at the center (Fig. 8, black triangle), and an average density of 40,000 neurons/ $\mathrm{mm}^{3}$ (Fig. 8) (Knox, 1982). We assumed that $80 \%$ of neurons were excitatory and $20 \%$ were inhibitory (Beaulieu et al., 1992) and that one-quarter of the inhibitory neurons were parvalbumin-positive in $\mathrm{S} 2 / 3$ (Xu et al., 2010). We then used the experimentally determined function describing the probability of connection of the $\mathrm{PV}+$ interneurons to the centrally located PC, which is an exponentially decaying function of distance with decay constant of 124 $\mu \mathrm{m}$ with no other adjustable parameters (Fig. 4E). We assumed that the probability of connection decayed similarly in every direction and chose the central PC as representative of any $\mathrm{S} 2 / 3 \mathrm{PC}$ in the neocortex.

When restricted to a $50-\mu$ m-thick slab centered on the PC, this model accurately replicated our results predicting that there would be six PV+ interneurons connected to the PC. Although in the maps we only observed an average of three connected $\mathrm{PV}+$ interneurons in $\mathrm{S} 2 / 3$, in this transgenic mouse only half the parvalbuminpositive interneurons are labeled (Chattopadhyaya et al., 2004).

We next extended the model to analyze the two implications posed at the beginning of this section, analyzing the full $500 \mu \mathrm{m}$ cube. Our model predicted there to be $46 \mathrm{PV}+$ interneurons connected to the PC. With the estimation of how many PV+ interneurons contact each PC, and the ratio of the numbers of PCs to PV + interneurons, we then calculated how many PCs each PV + interneuron contacts on average. For this calculation, we defined $C$ as the number of PCs, $V$ as the number of PV+ interneurons, $r=C / V$ as the ratio of PCs to $\mathrm{PV}+$ interneurons, $N$ as the number of PV+ interneurons that connect to each PC, $N$ • $C$ as the total number of $\mathrm{PV}+$ interneuron to $\mathrm{PC}$ connections, and $N \cdot C / V=N \cdot R$ as the number of PCs contacted by one PV+ interneuron.

Given that the number of PV + interneurons that connect to each PC (variable $N$ ) is 46 and that the ratio of PCs to PV+ interneurons (variable $R$ ) is $80 \%$ to $5 \%$ (see above paragraph), or 16 , the number of PCs contacted by one PV + interneuron is $N \cdot$ $r=46 \cdot 16=736$. It is known that PV + interneurons contact other PV + interneurons as well (Galarreta and Hestrin, 2002). Therefore, the total number of outgoing connections made by $\mathrm{PV}+$ interneurons is likely even higher. If we assume the same convergence of $46 \mathrm{PV}+$ interneurons onto one $\mathrm{PV}+$ as we found for PV + to PC connections, the number of outgoing PV + interneuron connections changes only from $N \cdot C$ to $N(C+V)$, such that the number of PCs and PV + interneurons contacted by one $\mathrm{PV}+$ interneuron algebraically reduces to $N(R+1)$, which is
$46 \cdot 17=782$. Therefore, we estimate that the typical PV + interneuron contacts $\sim 800$ postsynaptic neurons, both PCs and other PV+ interneurons.

Do PV + interneurons in somatosensory layer $2 / 3$ have enough boutons to contact 800 neurons? Fast-spiking (FS) basket cells in layer 2/3 have $\sim 4000$ boutons (Tamás et al., 1997; Wang et al., 2002; Karube et al., 2004). There are, on average, four boutons from PV+ interneurons on each postsynaptic soma at the age tested here (Chattopadhyaya et al., 2004). Therefore, we might expect $\sim 1000$ outgoing connections from PV+ interneurons.

A similar calculation was performed for the $\mathrm{S} 5$ and F2/3 datasets. We constructed a similar cube of neocortex for somatosensory layer 5 , in which the proportion of interneurons that are PV + is $\sim 60 \%$ ( $\mathrm{Xu}$ et al., 2010 ), and our experimentally determined spatial decay constant for connectivity was 183 $\mu \mathrm{m}$, resulted in $184 \mathrm{PV}+$ interneurons contacting each PC. As there are more $\mathrm{PV}+$ interneurons overall, the number of neurons contacted by each $\mathrm{PV}+$ interneuron was still $\sim 1000$ $[N(R+1)=184 \cdot(80 / 12+1)=1410]$. For frontal cortex layer $2 / 3$, the proportion of interneurons which are $\mathrm{PV}+$ is $\sim 40 \%$ (Xu et al., 2010) and the decay constant was $180 \mu \mathrm{m}$, resulting in $121 \mathrm{PV}+$ interneurons contacting each PC. The number of neurons contacted by each $\mathrm{PV}+$ interneuron was $121 \cdot(80 / 8+1)=1331$.

In summary, using a model, we estimate that the typical PV + interneuron in neocortex contacts several hundred to 1000 postsynaptic targets locally. At the same time, each PC is contacted by 
A

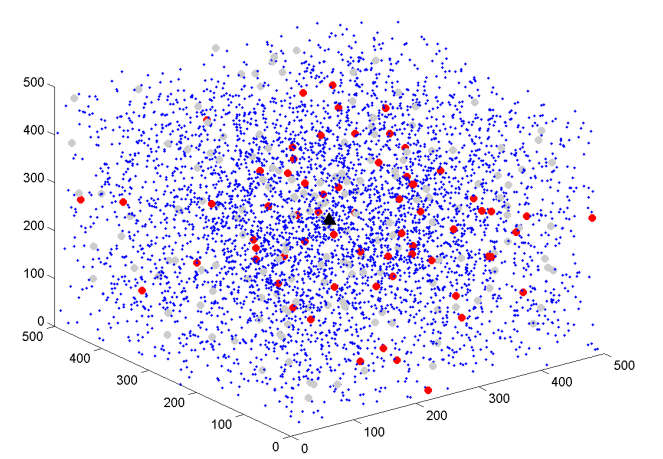

B
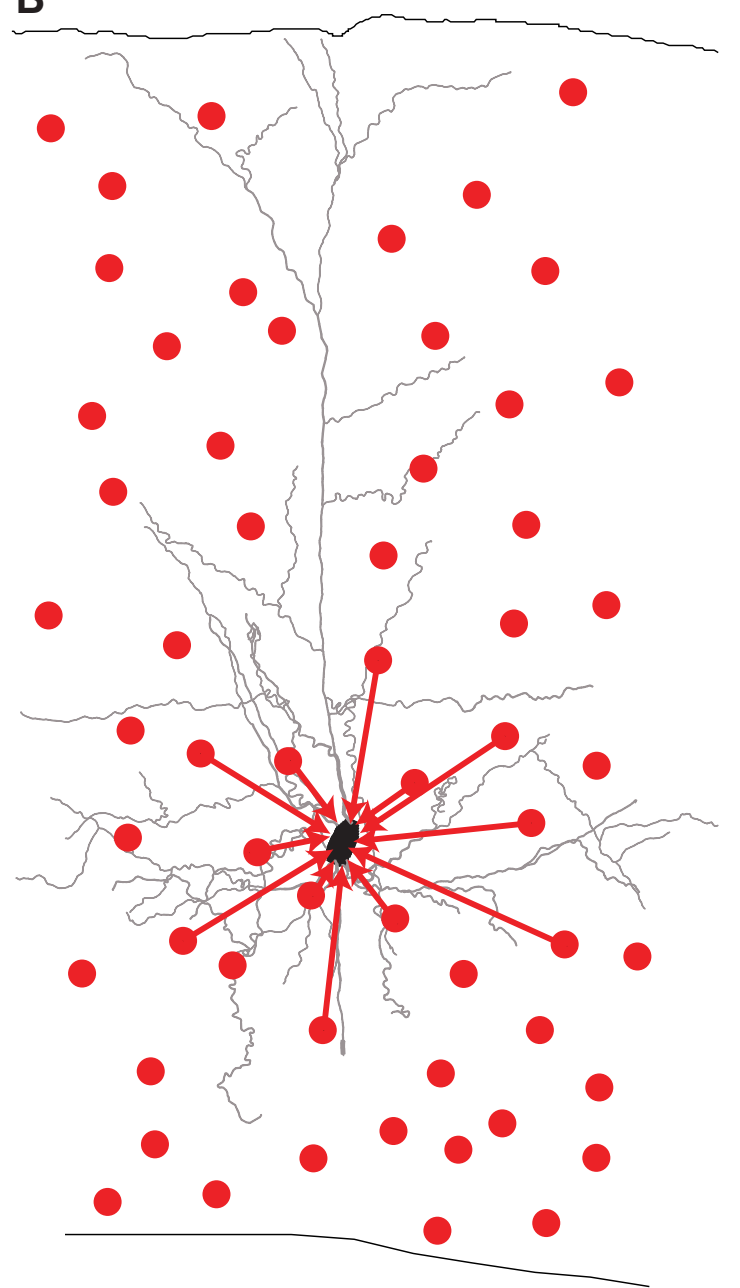

Figure 8. Model showing convergence of PV + interneurons onto one $P C . A, A$ cube of neocortex $500 \mu \mathrm{m}$ on each side from somatosensory layer $2 / 3$ with 5000 neurons. The cube was constructed with PV + interneurons and PCs using the connectivity profile we observed experimentally in S2/3, with one representative $\mathrm{PC}$ in black at the center. Other PCs are in blue, connected PV + interneurons in red, and unconnected PV + interneurons in gray. The density of connected PV + interneurons converging on this one $\mathrm{PC}$ can be visualized in three dimensions. $\boldsymbol{B}$, Schematic of the densely connected circuit architecture from PV + interneurons onto local PCS.

a large number of PV + interneurons, ranging from 50 to almost 200. Therefore, this PV+ interneuron-PC circuit has a high degree of local divergence and convergence.

Our results point to a circuit architecture in which PCs throughout neocortex and development receive a consistently high density of inputs from PV+ interneurons nearby (Fig. $8 B$ ).

\section{Discussion}

\section{Methodological considerations}

We used a novel method to map synaptic connections, so it is pertinent to discuss its advantages and potential limitations. This method relies on two-photon uncaging of glutamate to activate individual neurons while recording synaptic currents in other neurons, whose inputs we are attempting to map. Two-photon activation is critical to the spatial resolution of the technique as the small point-spread function produced by the two-photon process (Fig. 1 $H, I$ ) allows for single-cell accuracy.

Two potential problems with this optical technique are as follows: (1) failure to activate a targeted neuron and (2) inadvertent activation of a neuron which was not optically targeted. To ensure that the targeted neuron actually fired, we used different laser power levels, including a power that always fired PV + cells (300 $\mathrm{mW}$ on sample). Nevertheless, we cannot guarantee that every optically targeted neuron actually fired. Moreover, these experiments were performed in brain slices that have many severed inputs, so our strategy yields an underestimate of the true connectivity, implying that the density of connections may be higher than we observe.

To address the issue of inadvertently activating neurons, we tested whether PCs fired when the photostimulation was not centered on their somata. We rarely observed activation of a neuron when we targeted the laser outside its soma, even if we purposefully choose a PC with a lower rheobase than the PV+ interneurons for this calibration experiment (Fig. $1 \mathrm{~J}$ ). In addition, we used the switching test (Fig. $2 B$ ) to confirm the inhibitory identity of the photostimulated cell. Given the lower incidence of inhibitory interneurons relative to excitatory neurons in neocortex, it seems unlikely that another interneuron would be close enough to give rise to the inhibitory event observed.

Two independent, whole-cell recordings experiments validate the method and also confirm the results. First, predicted connections were confirmed by patching the targeted presynaptic interneuron (Fig. 2). Nine of 10 putatively connected pairs were indeed connected, while eight of eight putatively unconnected pairs were unconnected. Second, the optical results matched the dual whole-cell recordings from randomly selected PV + and PC pairs (Fig. 5)

\section{Dense connectivity of PV+ interneurons}

Our main result is a strikingly high density of connectivity from $\mathrm{PV}+$ interneurons onto nearby pyramidal cells in somatosensory cortex layer $2 / 3$ and 5 and frontal cortex layer $2 / 3$ (Figs. 4, 8). This is confirmed by dual whole-cell recordings (Fig. 5) and can approach, in some experiments, the limit of having every interneuron being connected to every local PC sampled. Although most data were taken at early developmental ages ( 2 weeks postnatal), we measured similar results in mature animals, so this dense connectivity is not a transient developmental stage (Fig. $4 H$ ).

Reported connectivity rates between interneurons identified as PV +, FS, or basket cells and nearby PCs vary greatly (Thomson et al., 1996, 2002; Tamás et al., 1997; Reyes et al., 1998; Wang et al., 2002; Holmgren et al., 2003; Yoshimura and Callaway, 2005; Kapfer et al., 2007; Thomson and Lamy, 2007; Oswald et al., 2009). Consistent with the high density we observed, connectivity rates of $67 \%$ have been reported for fast-spiking cells to PCs within $50 \mu \mathrm{m}$ in layer $2 / 3$ of rat somatosensory cortex (Kapfer et al., 2007). However, rates as low as $16 \%$ have been reported in layer 3 of rat cortex (Thomson et al., 2002). Intermediate values of 50-60\% have also been observed (Holmgren et al., 2003). In addition, a small decrease in FS to PC connectivity with interso- 
matic distance has been reported for layer $2 / 3$ of visual or somatosensory (Holmgren et al., 2003) and auditory (Oswald et al., 2009) cortices. Also, one-photon laser scanning studies report that inhibitory inputs coming from all interneuron subtypes arise from predominantly local sources (Dantzker and Callaway, 2000; Yoshimura et al., 2005) and have similar intralaminar patterns across areas (Kätzel et al., 2011).

We found $468 \mathrm{PV}+$ interneuron-to-PC connected pairs among 1747 pairs tested (not including false positives), a significant increase in the number of connections probed compared with past studies. These high numbers of tested pairs allowed us to draw conclusions about the basic rates of connectivity and to assess directly the true rates for each slice.

Using our large dataset, we calculated the function describing how the probability of connection varies with intersomatic distance. We found a high divergence from PV + interneurons outputs, which goes hand in hand with the convergence of many $\mathrm{PV}+$ interneurons onto one PC we observed in our highly dense maps. We estimate a higher density of converging connections onto PCs than previously calculated, even though our data may in fact underestimate the true probability of connection. Previous studies in neocortex (Tamás et al., 1997) and hippocampus (Buhl et al., 1994) estimated $\sim 25 \mathrm{PV}+$ interneurons converge onto one PC. This estimate is lower than what we found but it also predicts only $N(R+1)=25 \cdot 17=425$ connections from PV + interneurons to $\mathrm{PCs}$, which is low when one considers the large number of boutons present in a single PV+ axon ( 4000) (Wang et al., 2002; Karube et al., 2004). Therefore, the high density of connections we estimate from PV + interneurons to PCs appears more consistent with the observed high number of boutons.

We recently described how somatostatin-positive $(\mathrm{SOM}+)$ interneurons also make locally dense connections onto nearby PCs (Fino and Yuste, 2011). The average probability of connection from a SOM+ interneuron onto a PC in layer $2 / 3$ of frontal cortex was $43 \%$, somewhat higher than the average probability of connection we observed for PV + interneurons in any layer or area. But the probability of connection for $\mathrm{SOM}+$ interneurons within $200 \mu \mathrm{m}$ of a PC was $71 \%$, similar to the probabilities of connection we observed within $100 \mu \mathrm{m}$. The similarly dense matrix of local connections from these two distinct subpopulations of interneurons onto PCs implies that inhibitory connections may be built according to the same basic plan that, for both populations of neurons, also remains intact in mature animals.

\section{Unspecific innervation of PC cells}

Our second main result is the finding that the probability of two PCs receiving connections from the same $\mathrm{PV}+$ interneurons does not depend on whether the two PCs are synaptically connected (Fig. 6). This result is consistent with the high density of connections we observe as there is no room for specificity within such a high probability-in other words, there are no neurons to specifically avoid contacting.

In a past study, considering a pair of PCs that are synaptically connected as part of the same subcircuit revealed no difference in the inhibition received by the pair of PCs (Yoshimura et al., 2005). However, FS cells that were reciprocally connected to PCs shared more common input from excitatory sources than those that were not reciprocally connected (Yoshimura and Callaway, 2005). In addition, FS cells preferentially targeted PCs that provided reciprocal excitatory connections (Yoshimura and Callaway, 2005). Our method, with singlecell resolution, allowed us to directly determine whether PV+ interneuron connections to nearby PCs were specific. We found no evidence that $\mathrm{PV}+$ interneurons preferentially contact PC pairs in the same subcircuit (Fig. 6A). Our results therefore differ from those of Yoshimura and Callaway (2005), which showed preferential connections from FS cells to PCs providing reciprocal excitation. But, at the same time, we did not test the same hypothesis, as our optical strategy did not allow us to detect the response of the PV + interneurons to PC stimulation. In addition, the previous study assessed connectivity in the visual cortex of rats aged P21-P26, i.e., a different cortical area, age, and species than we used.

\section{A canonical microcircuit for neocortical inhibition?}

Our joint results, mapping the SOM+ (Fino and Yuste, 2011) and $\mathrm{PV}+$ (this study) interneurons demonstrate that inhibitory connections lack specificity in terms of contacting PCs. In addition, the broad inhibition provided by $\mathrm{PV}+$ interneurons and $\mathrm{SOM}+$ interneurons is consistent with recent findings that neurogliaform cells release GABA to mediate volume transmission to nearby neurons in an unspecific fashion (Oláh et al., 2009).

Consistent with this lack of specificity in their outputs, interneurons in layer $2 / 3$ of visual cortex are broadly tuned to the orientation of a stimulus (Sohya et al., 2007; Niell and Stryker, 2008; Kerlin et al., 2010; Ma et al., 2010; Zariwala et al., 2011), although some PV+ interneurons in the more superficial part of layer 2/3 appear to be sharply tuned (Runyan et al., 2010). Neocortical interneurons also receive input from neurons tuned to different orientations (Bock et al., 2011), providing an anatomical basis for the observation of broad tuning in interneurons.

Given that interneurons act as foreign invaders during development (Anderson et al., 1997), it is parsimonious that their wiring program may be relatively simple, without the need for mechanisms to specifically connect with existing pyramidal cell circuits. Extending this blanket of inhibition unspecifically over all local PCs (Fig. 8 B) could therefore represent a canonical circuit design for all inhibitory connections in the neocortex.

\section{References}

Aaron G, Yuste R (2006) Reverse optical probing (ROPING) of neocortical circuits. Synapse 60:437-440.

Anderson SA, Eisenstat DD, Shi L, Rubenstein JL (1997) Interneuron migration from basal forebrain to neocortex: dependence on Dlx genes. Science 278:474-476.

Ascoli GA, Alonso-Nanclares L, Anderson SA, Barrionuevo G, BenavidesPiccione R, Burkhalter A, Buzsáki G, Cauli B, Defelipe J, Fairén A, Feldmeyer D, Fishell G, Fregnac Y, Freund TF, Gardner D, Gardner EP, Goldberg JH, Helmstaedter M, Hestrin S, Karube F, et al. (2008) Petilla terminology: nomenclature of features of GABAergic interneurons of the cerebral cortex. Nat Rev Neurosci 9:557-568.

Beaulieu C, Kisvarday Z, Somogyi P, Cynader M, Cowey A (1992) Quantitative distribution of GABA-immunopositive and -immunonegative neurons and synapses in the monkey striate cortex (area 17). Cereb Cortex 2:295-309.

Berens P (2009) CircStat: A MATLAB toolbox for circular statistics. J Stat Soft 31:1-21.

Bock D, Lee WC, Kerlin A, Andermann ML, Soucy E, Yurgenson S, Wetzel AW, Hood G, Reid RC (2010) Local anatomical connectivity of a cluster of physiologically characterized cells in mouse visual cortex. Soc Neurosci Abstr 36:715.1

Bock DD, Lee WC, Kerlin AM, Andermann ML, Hood G, Wetzel AW, Yurgenson S, Soucy ER, Kim HS, Reid RC (2011) Network anatomy and in vivo physiology of visual cortical neurons. Nature 471:177-182.

Buhl EH, Halasy K, Somogyi P (1994) Diverse sources of hippocampal unitary inhibitory postsynaptic potentials and the number of synaptic release sites. Nature 368:823-828.

Callaway EM, Katz LC (1993) Photostimulation using caged glutamate re- 
veals functional circuitry in living brain slices. Proc Natl Acad Sci U S A 90:7661-7665.

Chattopadhyaya B, Di Cristo G, Higashiyama H, Knott GW, Kuhlman SJ, Welker E, Huang ZJ (2004) Experience and activity-dependent maturation of perisomatic GABAergic innervation in primary visual cortex during a postnatal critical period. J Neurosci 24:9598-9611.

Crick FH (1979) Thinking about the brain. Sci Am 241:219-232.

Cuntz H, Forstner F, Borst A, Häusser M (2010) One rule to grow them all: a general theory of neuronal branching and its practical application. PLoS Comput Biol 6:e1000877.

Dantzker JL, Callaway EM (2000) Laminar sources of synaptic input to cortical inhibitory interneurons and pyramidal neurons. Nat Neurosci 3:701-707.

Denk W, Horstmann H (2004) Serial block-face scanning electron microscopy to reconstruct three-dimensional tissue nanostructure. PLOS Biol 2:e329.

Douglas RJ, Martin KA (2004) Neuronal circuits of the neocortex. Annu Rev Neurosci 27:419-451.

Fino E, Yuste R (2011) Dense inhibitory connectivity in neocortex. Neuron 69:1188-1203.

Fino E, Araya R, Peterka DS, Salierno M, Etchenique R, Yuste R (2009) RuBi-Glutamate: two-photon and visible-light photoactivation of neurons and dendritic spines. Front Neural Circuits 3:2.

Galarreta M, Hestrin S (2002) Electrical and chemical synapses among parvalbumin fast-spiking GABAergic interneurons in adult mouse neocortex. Proc Natl Acad Sci U S A 99:12438-12443.

Holmgren C, Harkany T, Svennenfors B, Zilberter Y (2003) Pyramidal cell communication within local networks in layer $2 / 3$ of rat neocortex. J Physiol 551:139-153.

Kapfer C, Glickfeld LL, Atallah BV, Scanziani M (2007) Supralinear increase of recurrent inhibition during sparse activity in the somatosensory cortex. Nat Neurosci 10:743-753.

Karube F, Kubota Y, Kawaguchi Y (2004) Axon branching and synaptic bouton phenotypes in GABAergic nonpyramidal cell subtypes. J Neurosci 24:2853-2865.

Kätzel D, Zemelman BV, Buetfering C, Wölfel M, Miesenböck G (2011) The columnar and laminar organization of inhibitory connections to neocortical excitatory cells. Nat Neurosci 14:100-107.

Kerlin AM, Andermann ML, Berezovskii VK, Reid RC (2010) Broadly tuned response properties of diverse inhibitory neuron subtypes in mouse visual cortex. Neuron 67:858-871.

Knox CA (1982) Effects of aging and chronic arterial hypertension on the cell populations in the neocortex and archicortex of the rat. Acta Neuropathol 56:139-145.

Ma WP, Liu BH, Li YT, Huang ZJ, Zhang LI, Tao HW (2010) Visual representations by cortical somatostatin inhibitory neurons-selective but with weak and delayed responses. J Neurosci 30:14371-14379.

Markram H, Wang Y, Tsodyks M (1998) Differential signaling via the same axon of neocortical pyramidal neurons. Proc Natl Acad Sci USA 95:5323-5328.

Mountcastle VB (1982) An organization principle for cerebral function: the unit module and the distributed system. In: Mindful brain (Schmitt FO, ed), pp. 1-50. Cambridge, MA: MIT.

Niell CM, Stryker MP (2008) Highly selective receptive fields in mouse visual cortex. J Neurosci 28:7520-7536.

Nikolenko V, Poskanzer KE, Yuste R (2007) Two-photon photostimulation and imaging of neural circuits. Nat Methods 4:943-950.
Oláh S, Füle M, Komlósi G, Varga C, Báldi R, Barzó P, Tamás G (2009) Regulation of cortical microcircuits by unitary GABA-mediated volume transmission. Nature 461:1278-1281.

Oswald AM, Doiron B, Rinzel J, Reyes AD (2009) Spatial profile and differential recruitment of GABAB modulate oscillatory activity in auditory cortex. J Neurosci 29:10321-10334.

Peterlin ZA, Kozloski J, Mao BQ, Tsiola A, Yuste R (2000) Optical probing of neuronal circuits with calcium indicators. Proc Natl Acad Sci U S A 97:3619-3624.

Ramón y Cajal S (1923) Recuerdos de mi vida: historia de mi labor científica. Madrid: Alianza Editorial.

Reyes A, Lujan R, Rozov A, Burnashev N, Somogyi P, Sakmann B (1998) Target-cell-specific facilitation and depression in neocortical circuits. Nat Neurosci 1:279-285.

Runyan CA, Schummers J, Van Wart A, Kuhlman SJ, Wilson NR, Huang ZJ, Sur M (2010) Response features of parvalbumin-expressing interneurons suggest precise roles for subtypes of inhibition in visual cortex. Neuron 67:847-857.

Sohya K, Kameyama K, Yanagawa Y, Obata K, Tsumoto T (2007) GABAergic neurons are less selective to stimulus orientation than excitatory neurons in layer II/III of visual cortex, as revealed by in vivo functional Ca2+ imaging in transgenic mice. J Neurosci 27:2145-2149.

Tamás G, Buhl EH, Somogyi P (1997) Fast IPSPs elicited via multiple synaptic release sites by different types of GABAergic neurone in the cat visual cortex. J Physiol 500:715-738.

Thomson AM, Lamy C (2007) Functional maps of neocortical local circuitry. Front Neurosci 1:19-42.

Thomson AM, West DC, Hahn J, Deuchars J (1996) Single axon IPSPs elicited in pyramidal cells by three classes of interneurones in slices of rat neocortex. J Physiol 496:81-102.

Thomson AM, West DC, Wang Y, Bannister AP (2002) Synaptic connections and small circuits involving excitatory and inhibitory neurons in layers $2-5$ of adult rat and cat neocortex: triple intracellular recordings and biocytin labelling in vitro. Cereb Cortex 12:936-953.

Wang Y, Gupta A, Toledo-Rodriguez M, Wu CZ, Markram H (2002) Anatomical, physiological, molecular and circuit properties of nest basket cells in the developing somatosensory cortex. Cereb Cortex 12:395-410.

Wickersham IR, Lyon DC, Barnard RJ, Mori T, Finke S, Conzelmann KK, Young JA, Callaway EM (2007) Monosynaptic restriction of transsynaptic tracing from single, genetically targeted neurons. Neuron 53:639-647.

Woodruff A, Xu Q, Anderson SA, Yuste R (2009) Depolarizing effect of neocortical chandelier neurons. Front Neural Circuits 3:15.

Xu X, Roby KD, Callaway EM (2010) Immunochemical characterization of inhibitory mouse cortical neurons: three chemically distinct classes of inhibitory cells. J Comp Neurol 518:389-404.

Yoshimura Y, Callaway EM (2005) Fine-scale specificity of cortical networks depends on inhibitory cell type and connectivity. Nat Neurosci 8:1552-1559

Yoshimura Y, Dantzker JL, Callaway EM (2005) Excitatory cortical neurons form fine-scale functional networks. Nature 433:868-873.

Zariwala HA, Madisen L, Ahrens KF, Bernard A, Lein ES, Jones AR, Zeng H (2011) Visual tuning properties of genetically identified layer $2 / 3$ neuronal types in the primary visual cortex of cre-transgenic mice. Front Syst Neurosci 4:162. 